BNL-113152-2016-JA

\title{
High-precision measurement of magnetic penetration depth in superconducting films
}

X. He, A. Gozar, R. Sundling, and I. Božović

Submitted to Review of Scientific Instruments

November 2016

\author{
Condensed Matter Physics and Material Science Department \\ Brookhaven National Laboratory
}

\author{
U.S. Department of Energy \\ USDOE Office of Science (SC), \\ Basic Energy Sciences (BES) (SC-22)
}

Notice: This manuscript has been authored by employees of Brookhaven Science Associates, LLC under Contract No. DE-SC0012704 with the U.S. Department of Energy. The publisher by accepting the manuscript for publication acknowledges that the United States Government retains a non-exclusive, paid-up, irrevocable, world-wide license to publish or reproduce the published form of this manuscript, or allow others to do so, for United States Government purposes. 


\section{DISCLAIMER}

This report was prepared as an account of work sponsored by an agency of the United States Government. Neither the United States Government nor any agency thereof, nor any of their employees, nor any of their contractors, subcontractors, or their employees, makes any warranty, express or implied, or assumes any legal liability or responsibility for the accuracy, completeness, or any third party's use or the results of such use of any information, apparatus, product, or process disclosed, or represents that its use would not infringe privately owned rights. Reference herein to any specific commercial product, process, or service by trade name, trademark, manufacturer, or otherwise, does not necessarily constitute or imply its endorsement, recommendation, or favoring by the United States Government or any agency thereof or its contractors or subcontractors. The views and opinions of authors expressed herein do not necessarily state or reflect those of the United States Government or any agency thereof. 


\title{
High-precision measurement of magnetic penetration depth in super- conducting films
}

\author{
X. $\mathrm{He}^{1}$, A. Gozar ${ }^{1}$, R. Sundling ${ }^{2}$ and I. Božović ${ }^{1,3}$ \\ ${ }^{1}$ Yale University, Department of Applied Physics, New Haven CT 06511, USA \\ ${ }^{2}$ Zensoft Inc., Madison, WI 53705, USA \\ ${ }^{3}$ Brookhaven National Laboratory, Upton, New York 11973-5000, USA
}

The magnetic penetration depth $(\lambda)$ in thin superconducting films is usually measured by the mutual inductance technique. The accuracy of this method has been limited by uncertainties in the geometry of the solenoids and in the film position and thickness, by parasitic coupling between the coils, etc. Here, we present several improvements in the apparatus and the method. To ensure the precise thickness of the superconducting layer, we engineer the films at atomic level using atomic-layer-by-layer molecular beam epitaxy (ALL-MBE). In this way, we also eliminate secondary-phase precipitates, grain boundaries, and pinholes that are common with other deposition methods and that artificially increase the field transmission and thus the apparent $\lambda$. For better reproducibility, the thermal stability of our closed-cycle cryocooler used to control the temperature of the mutual inductance measurement has been significantly improved by inserting a custom-built thermal conductivity damper. Next, to minimize the uncertainties in the geometry we fused a pair of small yet precisely wound coils into a single sapphire block machined to a high precision. The sample is spring-loaded to exactly the same position with respect to the solenoids. Altogether, we can measure the absolute value of $\lambda$ with the accuracy better than $\pm 1 \%$.

\section{INTRODUCTION}

The magnetic penetration depth $\lambda$ is one of the key physical parameters characterizing a superconducting material. It is closely related to the superfluid phase stiffness (also called the helicity modulus) that determines how resilient the superconductor is to phase twists, and to the superfluid density. The temperature dependence $\lambda(T)$ encodes the information about the symmetry of the superconducting order parameter, showing for example whether it contains nodes, as is the case in unconventional superconductors ${ }^{1-3}$. Most commonly, $\lambda$ is measured using muon spin rotation $(\mu \mathrm{SR})^{4-10}$, microwave resonance (cavity-perturbation) ${ }^{11-13}$, or inductance techniques ${ }^{14-35}$.

A detailed comparison of various techniques used to measure the magnetic penetration depth in superconductors is beyond the scope of the present paper, and we refer the reader to several authoritative reviews on the subject, 
which contain hundreds of further references ${ }^{2,3,13,36}$. A short summary is as follows. Among the various techniques used, cavity-perturbation microwave measurements probably provide the highest precision. A self-oscillating tunneldiode resonator (TDR) can provide ${ }^{2}$ stable resolution of about 1 part per $10^{9}$. This means that relative changes of $\lambda$, such as e.g., the temperature dependence of $\lambda(T) / \lambda_{0}$, can be measured quite accurately; the resolution as high as $1-2$ $\AA$ can be achieved ${ }^{37}$. However, due to uncertainty in geometry, the absolute value $\lambda_{0}$ remains uncertain, and in fact is not even estimated directly in most microwave studies. In contrast, $\mu$ SR measures local magnetic fields, so it is not sensitive to the sample geometry and hence can give the absolute value of $\lambda$ with a reasonable (10\% or better) accuracy. However, $\mu$ SR experiments are slow and expensive, and hence typically limited to just a few samples and temperatures, which is not ideal for detailed studies of the dependence of $\lambda$ on temperature and doping. A reasonable compromise solution, implemented in a number of studies, is to use the microwave technique for highly accurate measurements of relative changes of $\lambda$ with temperature in a given sample, and then calibrate these data against the absolute value of $\lambda_{0}$ 'imported' from an independent study of the same material by another technique, most frequently $\mu$ SR (for a recent example, see e.g. Ref. 38). The accuracy of this procedure is limited by the absolute accuracy of the $\mu \mathrm{SR}$ technique itself, in particular because the analysis assumes a perfect vortex lattice while this assumption may not be fulfilled in real samples, and foremost because of potential differences between the samples used in different experiments. An alternative (and in principle more accurate) approach was introduced by Prozorov et al., ${ }^{39}$ who used the Al-coating technique that enables measuring the unknown penetration depth of the HTS material under study simultaneously with that of $\mathrm{Al}$; since the absolute value $\lambda_{0}$ in $\mathrm{Al}$ is known from the literature, it can be used to calibrate the results. The key uncertainty here is the quality and integrity of the Al film, which in practice may not turn out to be as prefect as the best Al single crystals used as the standard.

The two-coil mutual inductance method, in the transmittance geometry, is illustrated schematically In Fig. 1a. It was pioneered experimentally by A. Hebard and A. Fiory ${ }^{14}$, J. Claasen ${ }^{15}$, and T. Lemberger ${ }^{17,18}$, while the theoretical foundation was laid out by Clem and $\mathrm{Coffey}^{30}$. Consider a model superconducting film of infinite radius, thickness $d$, ac conductivity $\hat{\sigma}(\omega)=\sigma_{1}(\omega)-i \sigma_{2}(\omega)$, where $\omega$ is the measurement (angular) frequency, placed between two coils of radii $R_{1}$ and $R_{2}$, respectively, parallel to one another, and separated by a distance $D$. Then, the mutual inductance between the two coils is given by:

$$
\widehat{M}=R e M+i \operatorname{Im} M=\mu_{0} \pi R_{1} R_{2} \int_{0}^{\infty} d q \frac{e^{-q D} J_{1}\left(q R_{1}\right) J_{1}\left(q R_{2}\right)}{\cosh Q d+\left[\frac{\left(Q^{2}+q^{2}\right)}{2 q Q}\right] \sinh Q d}
$$

where $\mu_{0}=4 \pi \times 10^{-7} \mathrm{H} / \mathrm{m}, q$ is the wave-vector, $J_{l}(x)$ is the first-order Bessel function, and $Q^{2}=q^{2}+\frac{1}{\lambda^{2}}-i \mu_{0} \omega \sigma_{1}$. It is straightforward to generalize eq. (1) to calculate the mutual inductance between two solenoids with $N_{l}$ and $N_{2}$ turns, respectively, by performing the summation over every pair of coils, taking one from either side:

$$
\widehat{M}=\sum_{j=1}^{N_{1}} \sum_{k=1}^{N_{2}} M_{j, k}
$$

where $M_{j, k}$ is defined as in (1) except that now the distance $D$ should be replaced by $D_{j, k}$. Experimentally, one measures the inductance at a fixed frequency, varying the temperature, thus obtaining the $\operatorname{Re} M(T)$ and $\operatorname{Im} M(T)$ data files. Then, one can invert (numerically) eq. (2) to derive the corresponding functions $\lambda(T)$ and $\sigma_{I}(T)$. 
The accuracy of this procedure has some limitations ${ }^{32-35}$. First, one would expect the measured inductance to be somewhat larger, because films are finite and thus some magnetic flux 'leaks' around the film. It can also leak through the film if it has some pinholes or scratches, secondary-phase precipitates, or grain boundaries — all of which are quite commonly found in most films. In addition, there may be some other parasitic coupling between the two solenoids, through the electronics and the connections. Next, there are some uncertainties in the geometry - the shape and the size of the coils (usually home-made) and the position of the film with respect to the solenoids, which may vary run-to-run or as a function of temperature. These sources of errors have been analyzed in detail in the literature $^{32-35}$, and various solutions have been proposed and explored. Here we report a number of further improvements that significantly increase the accuracy of the measurement of $\lambda(T)$ dependence in thin superconducting films. The newly designed inductance measurement apparatus also simplifies the operation procedure, saving time and cost.

\section{MECHANICAL DESIGN}

To address the uncertainties in the geometry, minimize the eddy currents, and improve reproducibility, we have designed a device that incorporates the solenoids and the sample into a single sapphire crystal holder.

Solenoids. Both solenoids are wound using $0.025 \mathrm{~mm}$ oxygen-free copper (OFC) wire with insulation coating. The driving solenoid has 10 layers with the total of 300 turns; its inner diameter is $0.502 \mathrm{~mm}$, the outer diameter $1.050 \mathrm{~mm}$, the length $0.990 \mathrm{~mm}$, and the measured inductance is $30.7 \mu \mathrm{H}$. The pick-up coil has 30 layers with the total of 900 turns; its inner diameter is $1.010 \mathrm{~mm}$, the outer diameter $2.700 \mathrm{~mm}$, the length $0.980 \mathrm{~mm}$, and the measured inductance is $480 \mu \mathrm{H}$. The measured mutual inductance is $3.1 \mu \mathrm{H}$. The solenoids are manufactured (without a core or support) to a high precision by KUK Electronics AG in Appenzell, Switzerland. The driving coil is connected in series to a $10 \mathrm{k} \Omega$ resistor which is kept at room temperature. The resistance of the driving coil is $34.8 \Omega$ at room temperature, and it decreases as the sample is cooled down. The current in the driving coil is therefore essentially independent (to better than $0.3 \%$ ) on the temperature of the sample, but anyway it is measured directly. The resistance of the pick-up coil is $205.3 \Omega$ at room temperature, which is negligible in comparison to the voltage input impedance of the lock-in amplifier, which is $10 \mathrm{M} \Omega$. Nevertheless, we have verified this by measuring the temperature dependence of the real and imaginary part of the inductive voltage of pick-up coil. Our resolution is high enough that we can detect some small temperature dependence, but it is essentially negligible on the scale of the signal from the superconducting film. Still, it is included automatically in our calibration - the inductance of the film is normalized to the measured inductance of the pristine substrate.

Sapphire block. To hold the solenoids and the sample, a single-crystal sapphire block has been precisely machined to the accuracy of $0.0025 \mathrm{~mm}$ by Insaco, Inc. The cross-section is shown in Fig. 1b. Using G10 plastic and epoxy, the coils are fixed in concentric holes drilled in the sapphire block. Our standard substrate, of size $10 \times 10 \times 1$

$\mathrm{mm}^{3}$, fits into a slot that is $1.25 \mathrm{~mm}$ wide. Inside the slot, there is a machined wedge block made of G10 plastic. This block is mounted with two spring-loaded ('pogo') pins on one side so that the wedged side is in contact with one edge of the sample. Another wedge block and a door, both machined out of G10 plastic, hold the other side of the sample. By the virtue of the spring load, the two wedge blocks press on two upper edges of the sample without 
scratching the film surface, bringing the sample each time to the exactly same position. (The raw inductance measured in separate runs is reproducible to better than 0.3\%, as shown in Fig. 2 and detailed in Section III below.) The pick-up coil is $0.1 \mathrm{~mm}$ above the film surface. The drive coil is below, $0.50 \mathrm{~mm}$ away from the backside of the substrate. Both coils are aligned axially with the center of the sample. The uncertainty in the distance between the pickup coil and film $(0.1 \mathrm{~mm})$ and in the distance between drive-coil and backside of substrate $(0.50 \mathrm{~mm})$ it is about 0.01-0.02 $\mathrm{mm}$. The misalignment between the centers of the pick-up and drive coils is less than $0.1 \mathrm{~mm}$. However, these uncertainties do not generate a perceptible error in the measured value of $\lambda$. We normalize the measured inductance of the film by the inductance measured using a blank substrate. Since the geometry of the experiment does not change, in this way essentially all geometrical uncertainties are factored out.

Thermal connections. The bottom of the sample is in a close contact with the sapphire block to ensure good heat conduction. Sapphire provides excellent thermal conductivity (as good as copper) from $0.3 \mathrm{~K}$ to $300 \mathrm{~K}$ while providing good electric insulation. The sapphire block is thermally connected to the sample space of the cryocooler by a copper bracket, the shape of which can be seen in Fig. 1c. This particular cut ensures that there is no metal in the close proximity to the coil, in order to minimize the eddy currents.

One problem with the closed-cycle Gifford-McMahon (GM) cryocooler that we are using is that the piston motion causes temperature oscillations, the amplitude of which can reach up to $1 \mathrm{~K}$ for the temperature near $11 \mathrm{~K}$ where copper loses most of its thermal capacitance. This is illustrated in Fig. 2a, where the red line shows the temperature of the cold head upon cooling down from $T=57 \mathrm{~K}$ to $T=3.4 \mathrm{~K}$ at the rate of $0.1 \mathrm{~K} / \mathrm{min}$. To alleviate this problem, we have constructed ${ }^{40}$ a thermal damping system that consists of a combination of thin sheets of different materials (lead, stainless steel and copper) with contrasting thermal properties. This choice gives excellent results, more than satisfactory for our intended application (measurements of cryogenic transport and magnetic properties of high- $T_{\mathrm{c}}$ superconducting materials). From these metal sheets, we build a thermal buffer block, which we place between the cold head and the sample stage, as seen in the photograph of the actual device in Fig. 1d. This buffer acts as a lowpass filter for the heat transfer between the sample and the cold head; more details can be found in Ref. 40. In Fig. 2a, the black line shows the temperature of the sample space, which is evidently stabilized to better than $\delta T= \pm 1 \mathrm{mK}$ temperature, as seen in the inset of Fig. 2a. Since the substrates we use, $\mathrm{LaSrAlO}_{4}(\mathrm{LSAO})$ and $\mathrm{SrTiO}_{3}(\mathrm{STO})$, are poor heat conductors, we ramp the temperature at a slow rate $(0.1 \mathrm{~K} / \mathrm{min})$ to make sure that the sample reaches the desired temperature. Altogether, we achieve remarkable temperature stability, control, and reproducibility. This is illustrated in Figs. $2 \mathrm{~b}$ and $2 \mathrm{c}$ which shows the raw $\operatorname{Re} M(T)$ and $\operatorname{Im} M(T)$ data taken from the same sample twice, with the second measurement made 10 months later. The two results are almost identical; the variations are smaller than 1 $\mathrm{mK}$ for the temperature and less than $0.3 \%$ for the inductance (the absolute value is not very meaningful here because it depends on the frequency chosen).

Electrical connections. A plastic plate is fastened on top by brass screws to provide a platform for wiring and soldering. To minimize the electromagnetic interference while reducing the thermal losses, both the pick-up and the driving coil are connected by twisted pairs of 36AWG phosphor-bronze wire from the sample space to the cold head of the cryocooler. To further reduce noise and interference, the driving coil is connected to an oxygen-free copper 
(OFC) coaxial cable, while the pick-up coil is connected to a twin axial OFC cable with a silver shield. At the bottom, there is a coaxial feedthrough for the drive input and a triaxial feedthrough for the pick-up signal.

Outside the cryocooler, the driving signal is connected through a coaxial cable to a $10 \mathrm{k} \Omega$ resistor, and then connected further to the reference signal output of a Stanford Research Systems SR830 lock-in. With the $10 \mathrm{k} \Omega$ resistor outside of the cryocooler, the dependence of the driving current on the coil temperature is minimized, since the coil resistance is much smaller ( $34 \Omega$ at room temperature, decreasing to few $\Omega$ at low temperature). The pick-up signal is connected through a triaxial cable to the A and B signal inputs of the same lock-in amplifier. High quality triaxial and coaxial cables are used to further reduce noise and interference.

Vacuum. The inside of the cryocooler including the sample space is pumped by a Pfeiffer Hi-Cube oil free pumping station that includes a dry roughing pump and a 80 1/s turbo-molecular pump, reaching a high vacuum of $1 \times 10^{-6}$ Torr or better, within one hour.

\section{SYSTEM CALIBRATION}

To calibrate our inductance measurement system, we sputtered a $\mathrm{Nb}$ film at $T=500^{\circ} \mathrm{C}$ onto a $10 \times 10 \times 1 \mathrm{~mm}^{3}$ single-crystal $\mathrm{LaSrAlO}_{4}$ (LSAO) substrate manufactured by MTI Corporation. The substrate is polished perpendicular to the crystallographic [001] direction and is exactly the same as the substrates that we use for deposition of thin films of cuprate superconductors. This $\mathrm{Nb}$ film was $275 \mathrm{~nm}$ thick, which is enough to completely block magnetic field penetration once $\mathrm{Nb}$ is in the superconducting state. Figs. $2 \mathrm{~b}$ and $2 \mathrm{c}$ show the raw readings of the in-phase and out-of-phase outputs from the lock-in amplifier in our setup. The noise level of the raw data is less than $0.1 \%$. With the cooling rate of $0.1 \mathrm{~K} / \mathrm{min}$, we can resolve the sharp superconducting transition of the $\mathrm{Nb}$ film at $T_{c}=8.269 \mathrm{~K}$. The compound reproducibility in voltage and temperature is better than $0.3 \%$, on the scale of 10 months. The limiting factors here are the reproducibility of thermal loading and the rate at which the sample is cooled down.

Due to the finite size of the film, some flux 'leaks' around it even when the film is superconducting. To minimize this, we use inductance coils with a large number of turns $(300-1,500)$ but a very small inner radius $(250 \mu \mathrm{m})$, much smaller than the film size $\left(10 \times 10 \mathrm{~mm}^{2}\right)$, so that field leakage around the film is very small, less than $0.3 \%$, and this includes a small parasitic coupling through the electronics. Nevertheless, inductance measurements on $\mathrm{Nb}$ films allow us to accurately measure this small offset, since at the lowest temperature that we can reach with our cryocooler $\left(T=3.4 \mathrm{~K}\right.$, which is low enough compared to $\left.T_{c}=8.269 \mathrm{~K}\right)$ the field transmittance through a $275 \mathrm{~nm}$ thick $\mathrm{Nb}$ film is negligible. In Fig. $2 \mathrm{~b}$, the residual value $\Delta \operatorname{Re} V_{p}(T=3.5 \mathrm{~K})$ is the leakage of our system, about $0.3 \%$ of $\operatorname{Re} V_{p}\left(T>T_{c}\right)$. The residual value of $\Delta \operatorname{Im} V_{p}(T=3.5 \mathrm{~K}) \approx 0$, as expected.

As shown in Fig. 3a and $3 \mathrm{~b}, \operatorname{Re} V_{p}(T)$ and $\operatorname{Im} V_{p}(T)$ have some frequency dependence very near the superconducting transition but at lower temperatures this becomes negligible except for a small parallel upward shift in $\operatorname{Re} V_{p}(T)$ as the frequency is increased. As shown in Fig. 3c, the offset value $\Delta \operatorname{Re} V_{p}$ varies approximately linearly with frequency, for $20 \mathrm{kHz}<v<100 \mathrm{kHz}$. We have repeated this type of frequency-dependence studies on numerous films over 
several years, and verified that the data are indeed quite reproducible. We have done the same with thick films of $\mathrm{Pb}$ and $\mathrm{Al}$.

Another strict consistency check can be devised by noticing that $\lambda$, in contrast to $\Delta \operatorname{Re} V_{p}$, is an intensive quantity that (in this range) is frequency-independent. Hence, we can measure the inductance of a given film at several frequencies, subtract from $\operatorname{Re} V_{p}$ the corresponding offset $\Delta \operatorname{Re} V_{p}$, and test whether the inferred $\lambda$ is indeed independent on frequency. This procedure is illustrated in Fig. 4. Note that our measured mutual inductance $\widehat{M}\left(T>T_{c}\right)$ differs a little (typically by a couple percent) from the calculated one, due to some deviations in the geometry of the actual coil from its idealized mathematical model. To account for this (multiplicative) factor, we normalize the measured $\widehat{V}_{p}(T)$ by dividing it with its value $\widehat{V}_{p}^{\text {high }}$ just above $T_{c}$, or equivalently with $\widehat{V}_{p}^{\text {sub }}$ of a pristine substrate (the latter is almost independent on temperature and equal to $\widehat{V}_{p}^{\text {high }}$ ). Since the substrate is insulating, $\widehat{V}_{p}^{\text {sub }}=\operatorname{Re} \widehat{V}_{p}^{\text {sub. This nor- }}$ malization also factors out the scaling of the voltage on the pick-up coil (which is proportional to the impedance) with frequency. In Figs. $4 c$ and $4 d$, we plot the calibrated $\operatorname{Re} M(T) \equiv\left(\operatorname{Re} V_{p}-\Delta \operatorname{Re} V_{p}\right) / V_{p}^{\text {high }}$ and $\operatorname{Im} M(T) \equiv$ $\operatorname{Im} V_{p} / V_{p}^{\text {high }}$ curves, measured at three different representative frequencies, $v=20,40$, and $90 \mathrm{kHz}$. Apparently, the normalized curves essentially collapse onto one another, except very close to $T_{c}$ where one indeed expects some (small) difference because of the frequency dependence of $\hat{\sigma}(\omega, T)$ in the transition region.

We have also double-checked the validity of the above procedure using an alternative method, introduced previously by Lee $a t$ al. ${ }^{21}$ We deposit a thick layer of Al on top of the HTS film, and measure the mutual inductance of this composite bilayer. For these type of experiments, we use a similar mutual inductance setup, but within a He3based cryocooler capable of reaching $T=0.3 \mathrm{~K}$, and equipped with a $9 \mathrm{~T}$ superconducting magnet. The cuprate-Al bilayer shows two superconducting transitions, the first on due to the HTS films which only partially screens the field, resulting in non-zero transmittance even well below its $T_{c}$, and then the second one when $\mathrm{Al}$ becomes superconducting at $T_{c}=1.65 \mathrm{~K}$. Since $\mathrm{Al}$ is thick, at $T=0.3 \mathrm{~K}$ there is essentially no transmittance, and the measured signal is just the offset, $\Delta \operatorname{Re} V_{p}$. Then, we apply a small dc magnetic field (100 Gauss is sufficient), either perpendicular or parallel to the film surface, that drives Al normal but does not affect the HTS film. The transmittance jumps up, and the magnitude of this jump represents the accurate value of $\operatorname{Re} V_{p}(T=0.3 \mathrm{~K})$ of this HTS film, automatically corrected for the leakage offset $\Delta \operatorname{Re} V_{p}$. This method indeed gave the results identical to the one expounded above, to within a fraction of a percent. Note that this way of using Al coating is different from that of Prozorov et al. ${ }^{39}$; we do not need to know the actual value of $\lambda_{0}$ in the $\mathrm{Al}$ film, other than that it is much smaller than the thickness of the Al film so that the field transmission thorough $\mathrm{Al}$ at $T=0.3 \mathrm{~K}$ is negligible. We use $\mathrm{Al}$ just to shut-off the transmission through the sample and leave only the coupling around the film and through the electronics, revealing the true baseline.

Finally, we performed yet another check, as follows. First we measured $\operatorname{Re} V_{p}(T)$ and $\operatorname{Im} V_{p}(T)$ in a given film. Then we removed a $1 \mathrm{~mm}$-wide strip around the periphery of the film by chemical etching or ion-milling, thus reducing its size to a square of $9 \times 9 \mathrm{~mm}^{2}$ area, or alternatively patterning the film into to a disk of $9 \mathrm{~mm}$ diameter, then measured the inductance again. Then we cut the film down to $8 \mathrm{~mm}, 7 \mathrm{~mm}$, etc., measuring the inductance after each etching step. We have verified, in a number of films, that there was essentially no difference, within our targeted 
accuracy of $\pm 1 \%$, down to about $7 \mathrm{~mm}$ diameter. Hence, our standard $10 \times 10 \mathrm{~mm}^{2}$ films are large enough compared to the coils, and the field 'leakage' around the film is small enough, to justify the approximation made in deriving eq. (1).

\section{NUMERICAL INVERSION}

Once the accurate and well-calibrated experimental $\widehat{M}(T)$ data are obtained, the next step is to extract $\lambda(T)$ through numerical inversion. For easier data manipulation, we first bin the raw data and save the $\operatorname{Re} V_{p}(T)$ and $\operatorname{Im} V_{p}(T)$ as functions of temperature in $0.1 \mathrm{~K}$ steps; this is sufficiently accurate for our purpose, but could be made finer if needed. Then we subtract the respective offsets $\Delta \operatorname{Re} V_{p}$ and $\Delta \operatorname{Im} V_{p}$. Next, we normalize the data by dividing with $\operatorname{Re} V_{p}^{s u b}(T)$ and $\operatorname{Im} V_{p}^{s u b}(T)$, respectively; these are obtained from measurements on a pristine substrate, with the offsets properly removed, and binned in the same way. This provides the calibrated and normalized experimental $\operatorname{Re} M(T)$ and $\operatorname{Im} M(T)$ files. These data are compared to the tabulated array that maps a given pair of values $\left(\lambda, \sigma_{I}\right)$ to the corresponding pair $(\operatorname{Re} M, \operatorname{Im} M)$. The later values are calculated using eqs. (1) and (2), after the proper normalization which is achieved by dividing eq. (1) with:

$$
\widehat{M}^{h i g h}=\mu_{0} \pi R_{1} R_{2} \int_{0}^{\infty} e^{-q D} J_{1}\left(q R_{1}\right) J_{1}\left(q R_{2}\right) d q
$$

as appropriate for a very thin metallic film in the normal state. When $\operatorname{Im} V_{p}(T)$ drops below our noise floor (about 6 $\mathrm{nV}$ ) we set it to zero.

We note that computing eq. (2) over all $N_{1} \times N_{2}$ pairs of coils is computationally intensive. To calculate the full ( $\operatorname{Re} M, \operatorname{Im} M)$ to $\left(\lambda, \sigma_{1}\right)$ inversion array $\mathbf{M}_{1}$ in a reasonable time, we find the values $D_{j k}, R_{j}$, and $R_{k} / R_{j}$ for all pairs of coils $(j, k)$ and use Mathematica ${ }^{41}$ to construct a $10 \times 10 \times 10 \times 3$ array I as follows, with $p, q$, and $r$ from 1 to 10 :

$$
\begin{aligned}
& \mathrm{I}_{p q r 1}=\min D_{j k}+\frac{p}{10}\left(\max D_{j k}-\min D_{j k}\right) \\
& \mathrm{I}_{p q r 2}=\min R_{j}+\frac{q}{10}\left(\max R_{j}-\min R_{j}\right) \\
& \mathrm{I}_{p q r 3}=\min \frac{R_{k}}{R_{j}}+\frac{r}{10}\left(\max \frac{R_{k}}{R_{j}}-\min \frac{R_{k}}{R_{j}}\right)
\end{aligned}
$$

We note that eq. (1) is a continuous function between any two pairs of coils. Therefore, we use Mathematica's

Interpolation function to create a tricubic Hermite interpolation of the equation $f\left(\mathrm{I}_{p q r 1}, \mathrm{I}_{p q r 2}, \mathrm{I}_{p q r 3} \mathrm{I}_{p q r 2}\right)=\Upsilon_{p q r}$ over $p, q$ and $r$ from 1 to 10 , with $\Upsilon$ a new $10 \times 10 \times 10$ array which is left as a variable. The values of $f$ are presented to Interpolation at the discrete values of $\mathrm{I}$. The resulting function $f_{\text {interpolated }}\left(D, R_{1}, R_{2}\right)$ returns a linear combination of the entries of $\Upsilon_{p q r}$ for a given $D, R_{1}$, and $R_{2}$. We construct an $N_{1} \times N_{2} \times 10 \times 10 \times 10$ array $C$ of those coefficients for each pair of coils $(j, k)$, with $j$ from 1 to $N_{1}, k$ from 1 to $N_{2}$, and $p, q$ and $r$ from 1 to 10 : 


$$
C_{j k p q r}=f_{\text {interpolated }}\left(D_{j k}, R_{j}, R_{k}\right) \text { with } \Upsilon_{t u v}= \begin{cases}1, & \text { if } t u v=p q r \\ 0, & \text { otherwise }\end{cases}
$$

We use this to compute a $10 \times 10 \times 10$ array $\mathrm{H}$ which is the sum of coefficients across all pairs of coils, over $j$ from 1 to $N_{1}, k$ from 1 to $N_{2}$, and $p, q$ and $r$ from 1 to 10 :

$$
\mathrm{H}_{p q r}=\sum_{j, k} C_{j k p q r}
$$

We need to compute $\mathrm{H}$ only once for each geometry. We now create a $10 \times 10 \times 10$ array $\Psi$ using $\widehat{M}$ from eq. (1), over $p, q$, and $r$ from 1 to 10 :

$$
\Psi_{p q r}=\widehat{M} \text { with }\left\{\begin{array}{l}
D=\mathrm{I}_{p q r 1} \\
R_{1}=\mathrm{I}_{p q r 2} \\
R_{2}=\mathrm{I}_{p q r 3} \mathrm{I}_{p q r 2}
\end{array}\right.
$$

This allows us to represent an approximate solution to eq. (1) for any pair of coils $(j, k)$ as a linear combination of the values of $\Psi$ by taking the grand sum of the Hadamard (elementwise) product of $C_{j k}$ and $\Psi$ over $p, q$ and $r$ from 1 to 10 :

$$
\widehat{M}_{j k} \cong \sum_{p, q, r} C_{j k p q r} \Psi_{p q r}
$$

We need not compute these individual values, however, as we are only interested in their sum. Instead, we use $\mathrm{H}$ to arrive at an approximation of eq. (2), in a manner similar to eq. (8) approximating eq. (1), by taking the grand sum of the Hadamard product of $\mathrm{H}$ and $\Psi$ over $p, q$ and $r$ from 1 to 10 :

$$
\widehat{M} \cong \sum_{p, q, r} \mathrm{H}_{p q r} \Psi_{p q r}
$$

This allows us to compute an approximation of eq. (2) by computing only the $10^{3}$ integrals of $\Psi$ rather than all $N_{1} \times N_{2}$ integrals of eq. (2). For our particular geometry, this reduces the time required for the computations by a factor of 270 while the introduced error is less than one part in 3800 for Re $M$ and one part in 9000 for Im $M$. This approximation is performed entirely in the coil geometry space, without concern for any details of the integral itself beyond its continuity, but is sufficient for our desired precision.

To build the $(\operatorname{Re} M, \operatorname{Im} M)$ to $\left(\lambda, \sigma_{1}\right)$ inversion array, for each entry we utilize the gsl_integration_qags function of the GNU Scientific Library ${ }^{42}$, which applies the Gauss-Kronrod ${ }^{43}$ 21-point integration rule adaptively. We apply this to rapidly compute each of the $10^{3}$ integrals of $\Psi$. We then use this $\Psi$ in eq. (9). To fill the entire array, we compute approximately a half million pairs of $\left(\lambda_{m}, \sigma_{1 n}\right)$ with $1<m<601$ and $1<n<781$ with both $\lambda$ and $\sigma_{1}$ 
increasing in a geometric progression with minimum and maximum bounds chosen to be outside of our experimental values.

An inversion array $\mathbf{M}_{1}$ of this size gives 1.5 digits of precision in $\lambda$ and $\sigma_{1}$. To invert a particular value $M_{T}$ with greater precision, we use a recursive algorithm. We first locate the closest value to $M_{T}$ in $\mathbf{M}_{1}$ by weighted Cartesian distance. This gives an initial guess of $\widehat{g_{1}}=\left(\lambda_{m}, \sigma_{1 n}\right)$ where $m$ and $n$ are the column number and row number of the match in $\mathbf{M}_{1}$ and $\lambda_{m}$ and $\sigma_{1 n}$ are the corresponding $\lambda$ and $\sigma_{1}$ values. We let $\widehat{\lambda_{1}}=\left\langle\lambda_{m-1}, \lambda_{m}, \lambda_{m+1}\right\rangle$ and $\widehat{\sigma}_{1}=$ $\left(\sigma_{1_{n-1}}, \sigma_{1_{n}}, \sigma_{1_{n+1}}\right)$, then define $\widehat{\lambda_{2}}$ and $\widehat{\sigma_{2}}$ as follows, with $s, t=2$ :

$$
\begin{aligned}
& \widehat{\lambda_{k+1}}=\left(\widehat{\lambda}_{k_{s-1}}, \frac{\widehat{\lambda}_{k_{s-1}}+\widehat{\lambda}_{k_{s}}}{2}, \widehat{\lambda}_{k_{s}}, \frac{\widehat{\lambda}_{k_{s}}+\widehat{\lambda}_{k_{s}+1}}{2}, \widehat{\lambda}_{k_{s+1}}\right) \\
& \widehat{\sigma_{k+1}}=\left({\widehat{\sigma_{k}}}_{t-1}, \frac{{\widehat{\sigma_{k}}}_{t-1}+\widehat{\sigma}_{k_{t}}}{2},{\widehat{\sigma_{k}}}_{t}, \frac{{\widehat{\sigma_{k}}}_{t}+\widehat{\sigma}_{t+1}}{2}, \widehat{\sigma}_{t+1}\right)
\end{aligned}
$$

We construct a smaller $5 \times 5$ inversion array $\mathbf{M}_{2}$ from the values of $\widehat{\lambda_{2}}$ and $\widehat{\sigma_{2}}$, using the same procedure as calculating $\mathbf{M}_{1}$. We need not compute all twenty-five values, however, as we only need the nine values in the center, one of which has already been computed. We find the closest match $\widehat{g_{2}}$ to $M_{T}$ among these nine central values. $\widehat{g_{2}}$ has approximately $1 / 3$ of an additional digit of precision in $\lambda$ and $\sigma_{1}$ over $\widehat{g_{1}}$. We repeat to find $\widehat{g_{3}}$ : we let $s$ equal the column of $\widehat{g_{2}}$ in $\mathbf{M}_{2}$ and $t$ equal the row, calculate $\widehat{\lambda_{3}}$ and $\widehat{\sigma_{3}}$ using eq. (10), and compute $\mathbf{M}_{3}$ as we did $\mathbf{M}_{2}$. We continue iterating in this manner until we find $\widehat{g_{10}}$. These nine additional steps, requiring the calculation of just 72 additional values, produce an additional three digits of precision compared to $\widehat{g_{1}}$. This is sufficient for our needs, as it is beyond the level of precision of our integral approximation.

\section{A SOLUTION TO THE FILM THICKNESS PROBLEM: ATOMIC-LAYER ENGI- NEERING}

We have tested this system and procedure extensively by studying a large number of superconducting La $\mathrm{A}_{2-}$ ${ }_{\mathrm{x}} \mathrm{Sr}_{\mathrm{x}} \mathrm{CuO}_{4}$ (LSCO) films. For synthesis we used a unique atomic-layer-by-layer molecular beam epitaxy (ALL-MBE) system $^{44-47}$ Film growth was monitored in real time by reflection high-energy electron diffraction (RHEED). ALLMBE grown LSCO films are single-crystal, atomically smooth, and show no secondary-phase precipitates or pinholes $^{44-47}$. In Fig. 4, we show inductance data for an optimally doped ( $\left.\mathrm{x}=0.16\right)$ LSCO film. The film show a very sharp transition at $T_{c}=40.9 \mathrm{~K}$, testifying of its homogeneity. The half-width at half-maximum of the peak in $\operatorname{Im} M(T)$ (or the peak in $\operatorname{Re} \sigma(\omega)$, which is equally sharp) puts an upper limit $\Delta T_{c}=0.1 \mathrm{~K}$ on the variations in $T_{c}$ within the film of $10 \times 10 \mathrm{~mm}^{2}$ area.

The key remaining problem is uncertainty in the film thickness $d$ that must be entered as an input parameter in the equation (1). RHEED oscillations provide a digital count of atomic layers, while the lattice constant is accurately determined by X-ray diffraction. The problem with this is that a couple of layers next to the substrate and near the 
free film surface (if the film has been exposed to atmosphere) can be structurally and chemically modified to the extent that they are not superconducting. We alleviate this problem by atomic-layer engineering ${ }^{44-47}$. A five-unit-cells (5 UC) thick 'active' HTS layer was sandwiched between a protective buffer and a cover layer, both made out of metallic but non-superconducting $\left(M=\mathrm{La}_{1.60} \mathrm{Sr}_{0.40} \mathrm{CuO}_{4}\right)$. To minimize the interfacial effects (electron accumulation in $M$ and depletion in the nearest HTS layers ${ }^{45}$ ), $\mathrm{Sr}$ doping is graded near the interface. These transition layers are additionally doped by $3 \% \mathrm{Zn}$ (replacing $\mathrm{Cu}$ ) which greatly suppresses any residual interface superconductivity ${ }^{46}$. In this way we ensure that the superconducting layer is exactly 5 UC thick.

To test the validity of this approach, we have synthesized a series of LSCO films with the composition of the constituent materials unaltered, but with the thickness of the active HTS layers varied by over two orders of magnitude, from $0.66 \mathrm{~nm}$ (one-half unit cell thick) to over $100 \mathrm{~nm}$. We have verified (see Extended data Fig. 1 in Ref. 47) that the sheet superfluid density scaled linearly with thickness, while the inferred $\lambda(T)$ dependence was independent on thickness, as one would expect. This additionally validates the accuracy of the method, as well as testifying on the film quality including absence of pinholes, macroscopic defects, grain boundaries, etc. Still, we emphasize that to get the accurate absolute value of $\lambda$, this last step - determining the superconducting film thickness with atomic-layer precision - is crucial. This is the main source of error in determining the absolute value of $\lambda$, once we have tightened the error bars on reproducibility by improvements in the hardware and in the ways to correct for the parasitic coupling. Note that in general the thickness of the superfluid layer need not be equal to the film thickness (which itself is also known only to within some error), because of superfluid-depleted layers near the surface and near the substrate. To minimize this error, we use ALL-MBE and sophisticated sample engineering at the atomic-layer level. But this still leaves us with the accuracy of about $1 \%$, at best. This seems hard to improve further with the synthesis and characterization techniques available today.

In Fig. 5, we show examples of $\lambda(T)$ dependences derived from inductance data for several LSCO films. In Figs. $5 \mathrm{a}$ and $5 \mathrm{~b}$, the only data processing was (i) subtraction of the offset (which is very small anyway), (ii) normalization

of the measured $\widehat{V}_{p}(T)$ by dividing it with the measured $V_{p}^{\text {high }}$ and (iii) binning the data in steps of $0.1 \mathrm{~K}$ to make the file size tractable (one raw data file as collected contains $\sim 10^{5} \widehat{V}_{p}(T)$ data points). In Ref. 47, similar data are shown for a set of 100 LSCO films covering the entire overdoped side of the LSCO phase diagram in very fine steps in chemical composition. In these experiments, we chose a driving current of $I_{\mathrm{d}}=40 \mu \mathrm{A}$. With this current, the magnetic field generated by the driving coil and measured at the position of the sample with a magnetometer is less than 0.1 Gauss. This is much smaller than the lower critical field in LSCO, so it cannot induce vortices or reduce $T_{c}$ in our superconducting films.

\section{CONCLUSION}

We have built a next-generation mutual-inductance setup that features a novel single sapphire block design with fixed, small, precise solenoids coils and spring-loaded sample mounting, ensuring accurate and reproducible sample positioning. Flux leakage and parasitic coupling is minimized, measured accurately, and corrected for. Superconduct- 
ing films are engineered at atomic-layer level in order to achieve accurate thickness control, and an accurate numerical inversion procedure is implemented. Altogether, the accuracy in measuring the absolute value of $\lambda$ has been improved to reach $\pm 1 \%$. The setup is based on a closed-cycle cryocooler, so that the running costs are low. Sample loading is easy and quick, and the temperature control very precise (to $\pm 1 \mathrm{mK}$ ) and automatic. We have been using one mutual-inductance apparatus such as described here in the $0.3 \mathrm{~K}<T<300 \mathrm{~K}$ temperature range in the Helium-3 system, and two more in the $3.5 \mathrm{~K}<T<300 \mathrm{~K}$ range in a closed-cycle cryocooler. With this equipment, in the last couple years we have measured more than 1,000 samples. The results are very reproducible, to better than $\pm 0.3 \%$ (see Figures $2 \mathrm{~b}$ and $2 \mathrm{c}$ ), and the apparatus is robust, reliable, and easy to operate; we had no mechanical or electrical failures whatsoever. One cautionary measure we take is that we keep the cooling and heating rates of the apparatus below $3 \mathrm{~K} / \mathrm{min}$, in order to reduce thermally induced stress on the apparatus. This is a valuable tool to study superconductors, from routine characterization (such as measuring $T_{c}$ ) to detailed basic studies ${ }^{47}$ of the dependence of superfluid phase stiffness (the helicity modulus) and the superfluid density on temperature, chemical composition and doping, epitaxial strain, etc.

\section{ACKNOWLEDGEMENTS}

We are grateful to $\mathrm{T}$. Lemberger for a careful reading and useful comments. This research done at BNL and was supported by the U.S. Department of Energy, Basic Energy Sciences, Materials Sciences and Engineering Division. X.H. and A.G. are supported by the Gordon and Betty Moore Foundation.

\section{References}

1. M. Tinkham, Introduction to superconductivity, 2nd ed., (McGraw Hill, 1996)

2. R. Prozorov and R. W. Giannetta, Supercond. Sci. Tech. 19, R41 (2006).

3. R. Prozorov and V. G. Kogan, Rep. Prog. Phys. 74, 124505 (2011).

4. Y. J. Uemura, G. M. Luke, B. J. Sternlieb, J. H. Brewer, J. F. Carolan, W. N. Hardy, R. Kadono, J. R. Kempton, R. F. Kiefl, S. R. Kreitzman, P. Mulhern, T. M. Riseman, D. Ll. Williams, B. X. Yang, S. Uchida, H. Takagi, J. Gopalakrishnan, A. W. Sleight, M. A. Subramanian, C. L. Chien, M. Z. Cieplak, G. Xiao, V. Y. Lee, B. W. Statt, C. E. Stronach, W. J. Kossler, and X. H. Yu, Phys. Rev. Lett. 62, 2317 (1989).

5. Y. J. Uemura, A. Keren, L. P. Le, G. M. Luke, W. D. Wu, Y. Kubo, T. Manako, Y. Shimakawa, M. Subramanian, J. L. Cobb and J. T. Markert, Nature (London) 364, 605 (1993).

6. Ch. Niedermayer, C. Bernhard, U. Binninger, H. Glückler, J. L. Tallon, E. J. Ansaldo, and J. I. Budnick, Phys. Rev. Lett. 71, 1764 (1993).

7. C. Bernhard, Ch. Niedermayer, U. Binninger, A. Hofer, Ch. Wenger, J. L. Tallon, G. V. M. Williams, E. J. Ansaldo, J. I. Budnick, C. E. Stronach, D. R. Noakes, and M. A. Blankson-Mills, Phys. Rev. B 52, 10488 (1995).

8. C. Panagopoulos, T. Xiang, W. Anukool, J. R. Cooper, Y. S. Wang, and C. W. Chu, Phys. Rev. B 67, 220502(R) (2003). 
9. J. L. Tallon, J. W. Loram, J. R. Cooper, C. Panagopoulos, and C. Bernhard, Phys. Rev. B 68, 180501(R) (2003).

10. E. Morenzoni, B. M. Wojek, A. Suter, T. Prokscha, G. Logvenov, and I. Božović, Nature Commun. 2, 272 (2011).

11. A. Hosseini, R. Harris, S. Kamal, P. Dosanjh, J. Preston, R. Liang, W. N. Hardy, and D. A. Bonn, Phys. Rev. B 60, 1349 (1999).

12. D. M. Broun, W. A. Huttema, P. J. Turner, S. Özcan, B. Morgan, R. Liang, W. N. Hardy, and D. A. Bonn, Phys. Rev. Lett. 99, 237003 (2007).

13. D. A. Bonn and W. N. Hardy, In Physical Properties of High Temperature Superconductors V, edited by D. M. Ginsberg (World Scientific, Singapore, 1996) pp. 7-98.

14. A. F. Hebard, and A. T. Fiory, Phys. Rev. Lett. 44, 291 (1980).

15. J. H. Claassen, M. E. Reeves, and R. J. Soulen Jr., Rev. Sci. Instrum. 62, 996 (1991).

16. J.-P. Locquet, Y. Jaccard, A. Cretton, E. J. Williams, F. Arrouy, E. Mächler, T. Schneider, O. Fischer, and P. Martinoli, Phys. Rev. B 54, 7481 (1996).

17. T. R. Lemberger, I. Hetel, A. Tsukada, M. Naito, and M. Randeria, Phys. Rev. B 83, 140507 (2011).

18. J. Y. Lee and T. R. Lemberger, Appl. Phys. Lett. 62, 2419 (1993).

19. A. Fuchs, W. Prusseit, P. Berberich, and H. Kinder, Phys. Rev. B. 53, 14745 (1996).

20. J. Gilchrist, and E. H. Brandt, Phys. Rev. B 54, 3530 (1996).

21. J. Y. Lee, Y. H. Kim, T-S. Hahn, and S. S. Choi, Appl. Phys. Lett. 69, 1637 (1996).

22. J. H. Claassen, M. L. Wilson, J. M. Byers, and S. Adrian, J. Appl. Phys. 82, 3028 (1997).

23. K. M. Paget, S. Guha, M. Z. Cieplak, I. E. Trofimov, S. J. Turneaure, and T. R. Lemberger Phys. Rev. B. 59, 641 (1999).

24. R. F. Wang, S. P. Zhao, G. H. Chen, and Q. S. Yang, Appl. Phys. Lett. 75, 3865 (1999).

25. A. Rufenacht, J. P. Locquet, J. Fompeyrine, D. Caimi, and P. Martinoli, Phys. Rev. Lett. 96, 227002 (2006).

26. T. R. Lemberger, I. Hetel, A. Tsukada, and M. Naito, Phys. Rev. B 82, 214513 (2010).

27. A. Gauzzi, J. Le Cochec, G. Lamura, B. J. Jönsson, V. A. Gasparov, F. R. Ladan, B. Plaçais, P. A. Probst, D. Pavuna and J. Bok, Rev. Sci. Instrum. 71, 2147 (2000).

28. V. A. Gasparov, and I. Bozovic, Phys. Rev. B 86, 094523 (2012).

29. M. Došlić, D. Pelc, and M. Požek, Rev. Sci. Instrum. 85, 073905 (2014).

30. J. R. Clem, and M. W. Coffey, Phys. Rev. B 46, 14662 (1992).

31. A. B. Pippard, Supercond, Sci. Tech. 7, 696 (1994).

32. S. J. Turneaure, E. R. Ulm, and T. R. Lemberger, J. Appl. Phys. 79, 4221 (1996).

33. S. J. Turneaure, A. A. Pesetski, and T. R. Lemberger, J. Appl. Phys. 83, 4334 (1998).

34. M. W. Coffey, J. Appl. Phys. 87, 4344 (2000).

35. M. W. Coffey, J. Appl. Phys. 89, 5570 (2001).

36. D. N. Basov, and T. Timusk, Mod. Phys. 77, 721 (2005).

37. W. N. Hardy, D. A. Bonn, D. C. Morgan, R. X. Liang, and K. Zhang, Phys. Rev. Lett. 70, 3999 (1993). 
38. O. Ofer, J. C. Baglo, M. D. Hossain, R. F. Kiefl, W. N. Hardy, A. Thaler, H. Kim, M. A. Tanatar, P. C. Canfield, R. Prozorov, G. M. Luke, E. Morenzoni, H. Saadaoui, A. Suter, T. Prokscha, B. M. Wojek, and Z. Salman. Phys. Rev. B 85, 060506 (2012).

39. R. Prozorov, R. W. Giannetta, A. Carrington, P. Fournier, R. L Greene, P. Guptasarma, D. G. Hinks, A. R. Banks, Appl. Phys. Lett. 77, 4202 (2000).

40. G. Dubuis, X. He, and I. Božović, Rev. Sci. Instrum. 85, 103902 (2014).

41. Wolfram Research, Inc., Mathematica, Version 9.0, (Champaign, IL, 2012).

42. M. Galassi et al, GNU Scientific Library Reference Manual (3rd Ed.), ISBN 0954612078 (2009).

43. D. Kahaner, C. Moler, S. Nash, Numerical Methods and Software (Prentice-Hall, 1989). ISBN 978-0-13627258-8

44. I. Bozovic, IEEE Trans. Appl. Supercond. 11, 2686 (2001).

45. A. Gozar, G. Logvenov, L. Fitting Kourkoutis, A. T. Bollinger, L. A. Giannuzzi, D. A. Muller, and I. Bozovic, Nature 455, 782 (2008).

46. G. Logvenov, A. Gozar, A. and I. Bozovic, Science 326, 699 (2009).

47. I. Božović, X. He, J. Wu and A. T. Bollinger, Nature 536, 309 (2016). 
(a)

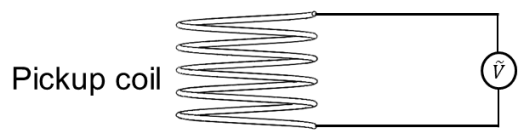

Film

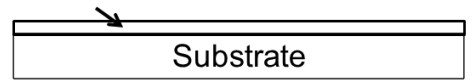

Drive coil

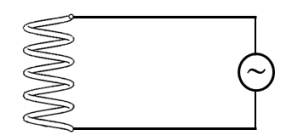

(c)

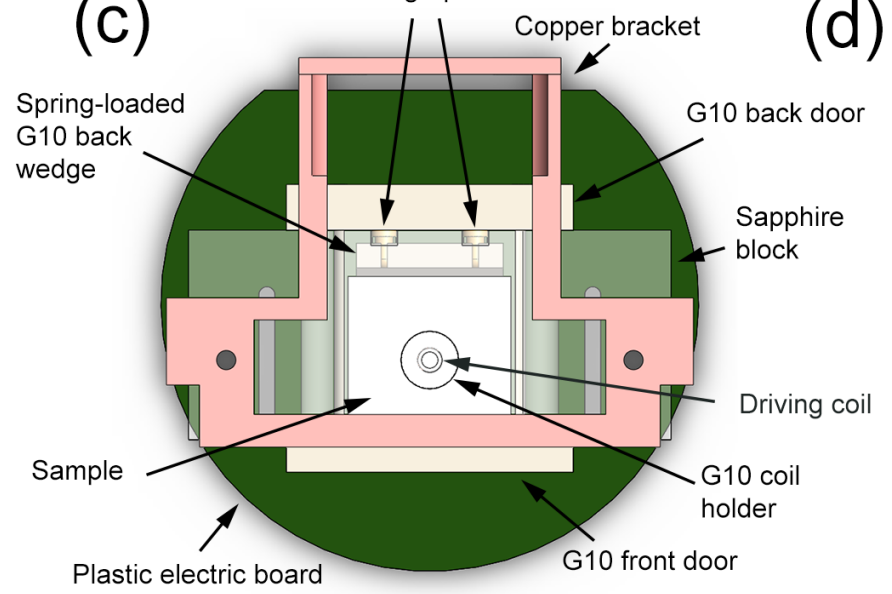

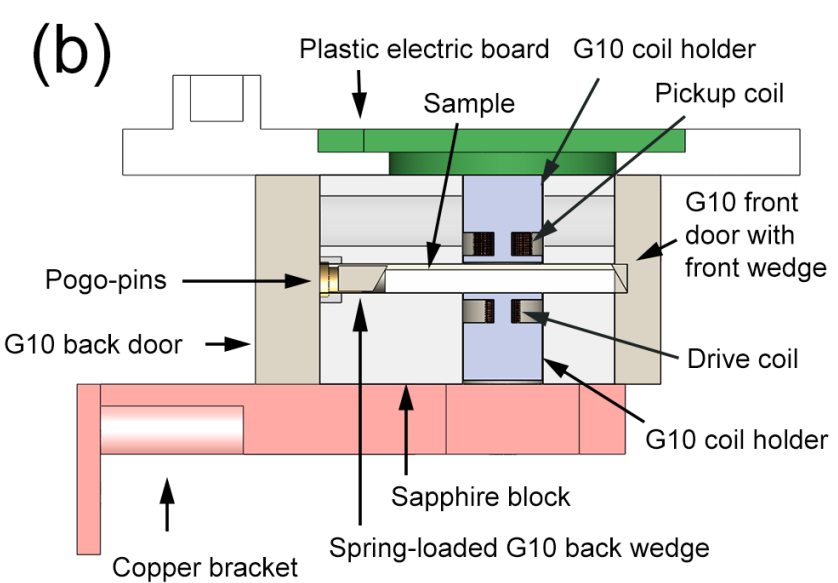

(d)

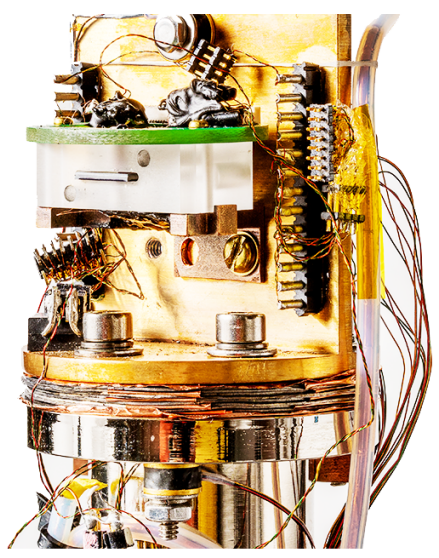

FIG. 1. (a) A schematics of the mutual inductance (MI) device used in this research. A 300-turn driving coil is connected to the reference output signal of a lock-in amplifier. A 900-turn pick-up coil is connected to the signal input of the same amplifier. The substrate is sandwiched between the two coils with the film facing the pick-up coil. (b) The cross-section view of the MI device. The back of the sample rests on the bottom of the slot carved inside the sapphire block. A spring-loaded wedge inside the slot and a wedge on the door clamp the sample down so that the backside of the substrate is firmly pressed onto the sapphire surface. The springs are contained inside the pogo-pins, which are glued to the wedge. The distance between the film surface and the pick-up coil is $0.4 \mathrm{~mm}$. The sapphire block is tightened down to a copper bracket connecting it to the sample space. (c) The bottom view of the MI device, showing that the copper bracket is carved out in the middle so that near the coil there is no metal where eddy currents could be induced. (d) A photograph of the MI device mounted on the sample space of the cryocooler. The door of the device is open to show the mounting position of the sample inside the sapphire block. The MI device is fastened onto a gold-plated copper disk for thermal contact. Below this disk is our thermal damping system that consists of a series of thin sheets of lead, stainless steel and copper ${ }^{40}$. One thermocouple is connected to the sample space and another to the cold head of the cryocooler. 

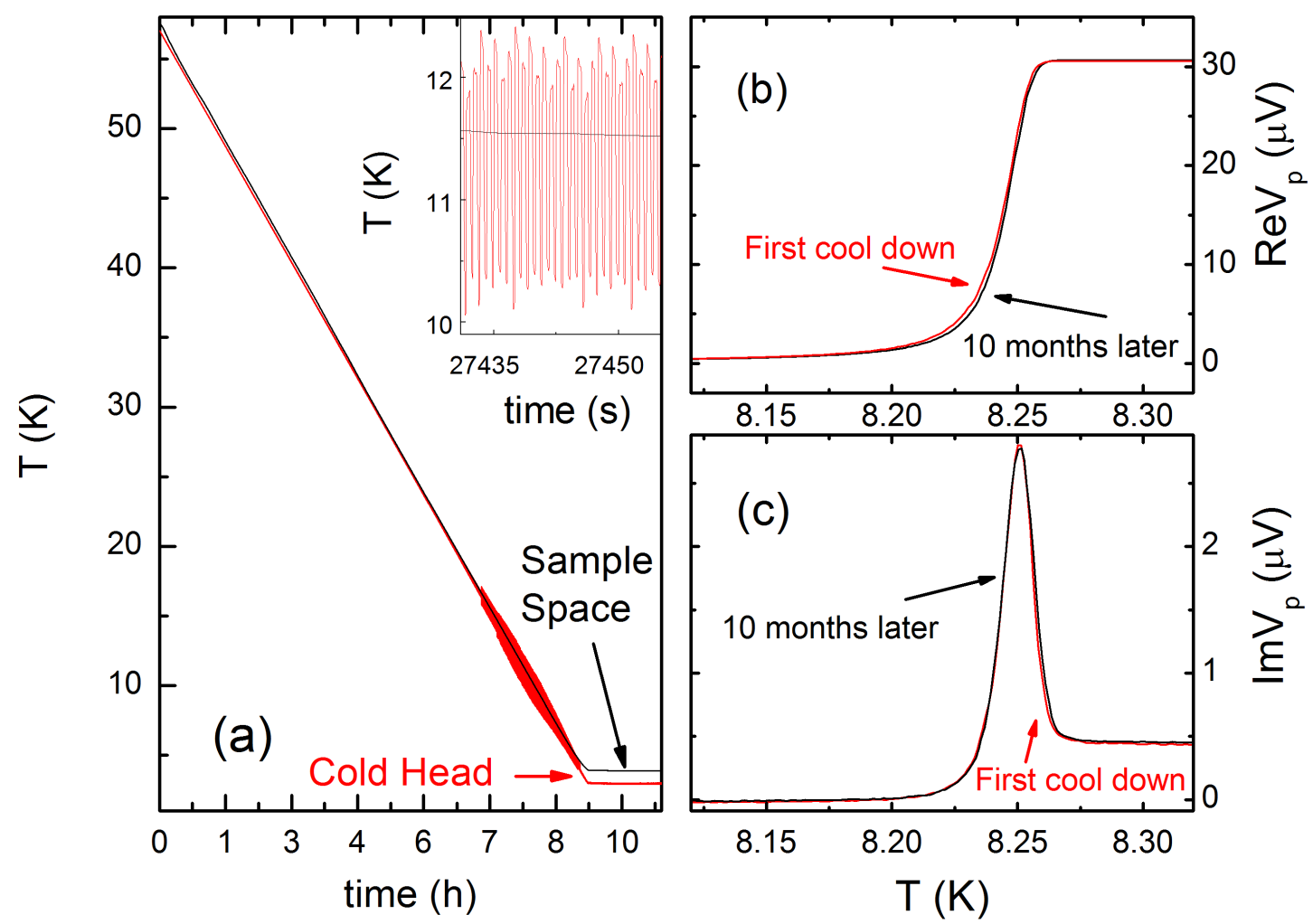

FIG. 2. (a) The temperature of the cold head (the red line) and of the sample space (the black line) upon cooling from $60 \mathrm{~K}$ to $3.4 \mathrm{~K}$ at a constant rate of $0.1 \mathrm{~K} / \mathrm{min}$. The inset: a zoomed-in view of the raw temperature data showing that the amplitude of oscillation of the cold-head temperature reaches $1 \mathrm{~K}$ while the sample space is stabilized to better than $1 \mathrm{mK}$. This is achieved by virtue of a custom multilayer spacer ${ }^{40}$ that provides excellent thermal damping together with sufficient thermal conductivity to reach the base temperature of $3.4 \mathrm{~K}$. (b) The raw $\operatorname{Re} V_{p}(T)$ data taken from a $\mathrm{Nb}$ film grown on LSAO substrate measured on 03/22/2015 (the red line) and ten months later, on 01/20/2016 (the black line). (c) The corresponding $\operatorname{Im} V_{p}(T)$ data. On the one-year scale, the reproducibility in both $\operatorname{Re} V_{p}(T)$ and $\operatorname{Im} V_{p}(T)$ is better than $0.3 \%$. 

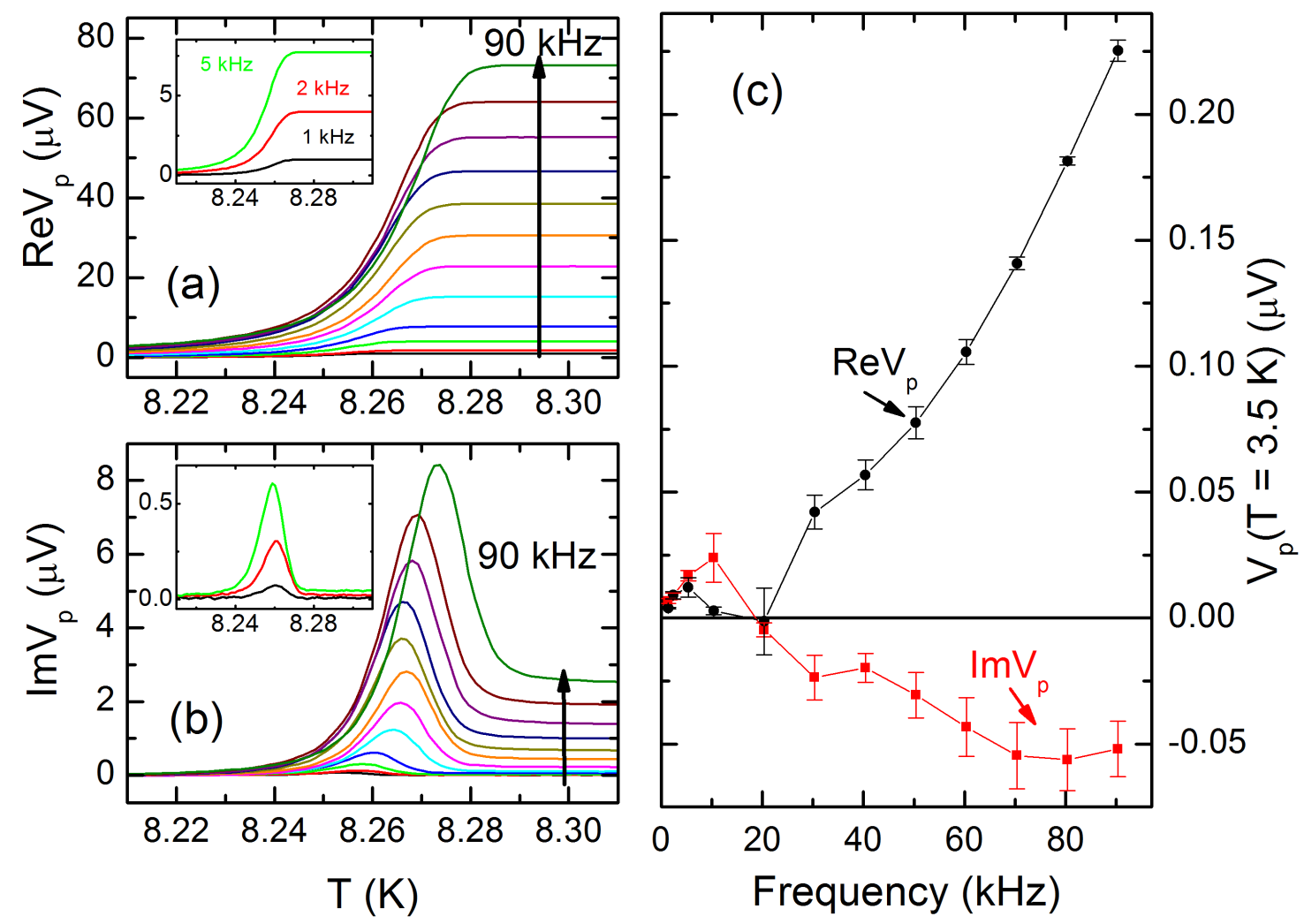

FIG 3. (a) The raw $\operatorname{Re} V_{p}(T)$ data from a $\mathrm{Nb}$ film grown on an LSAO substrate, measured at various frequencies: 1 kHz, $2 \mathrm{kHz}, 5 \mathrm{kHz}, 10 \mathrm{kHz}, 20 \mathrm{kHz}, 30 \mathrm{kHz}, 40 \mathrm{kHz}, 50 \mathrm{kHz}, 60 \mathrm{kHz}, 70 \mathrm{kHz}, 80 \mathrm{kHz}$, and $90 \mathrm{kHz}$. (b) The corresponding $\operatorname{Im} V_{p}(T)$ data. (c). Black circles: $\operatorname{Re} V_{p}(T=3.5 \mathrm{~K})$ as a function of frequency; red squares: $\operatorname{Im} V_{p}(T=3.5 \mathrm{~K})$. All these measurements were done using the drive current of the same amplitude, $I_{d}=40 \mu \mathrm{A}$. 

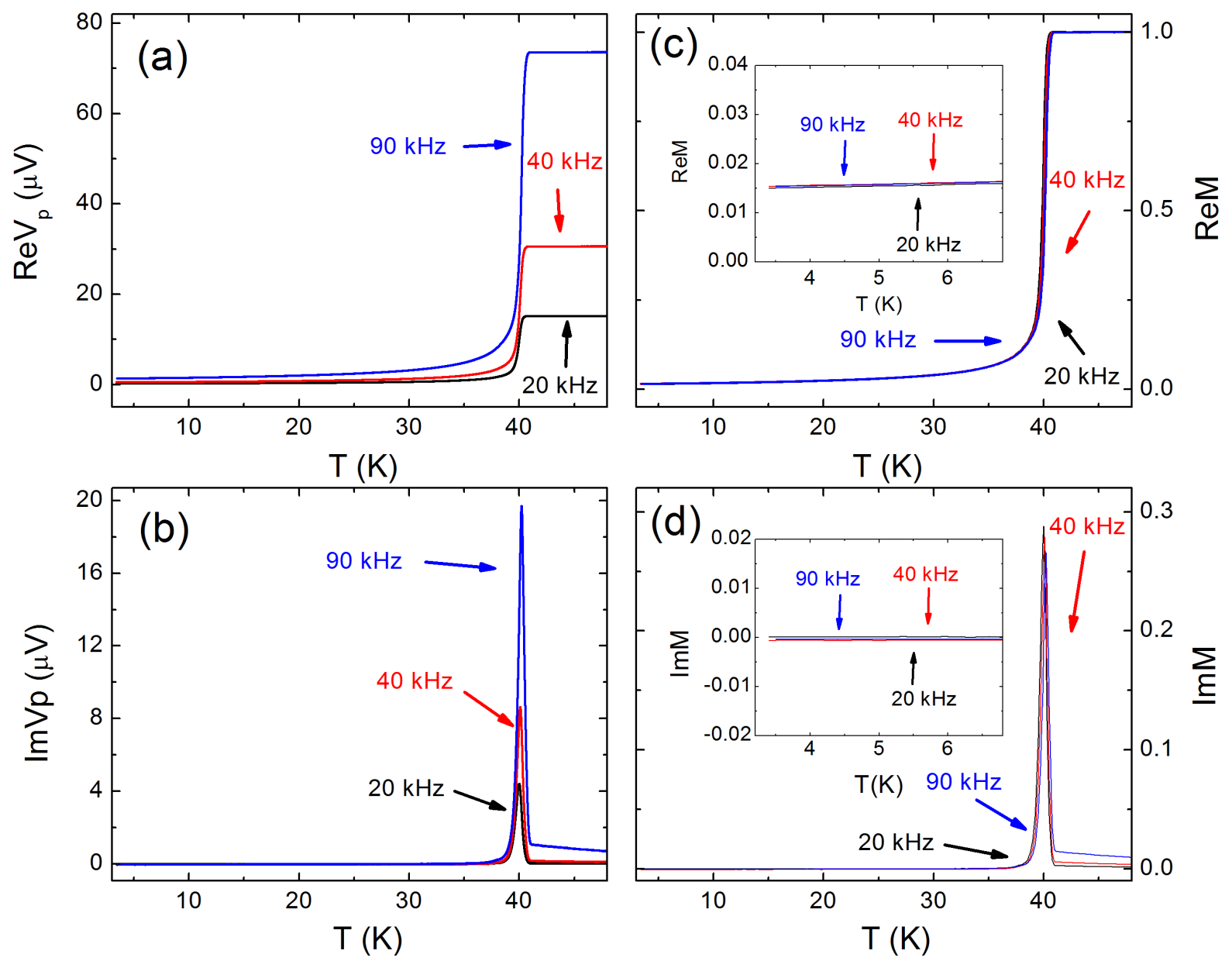

FIG. 4. (a) The raw $\operatorname{Re} V_{p}(T)$ data from an optimally-doped $\mathrm{La}_{1.84} \mathrm{Sr}_{0.16} \mathrm{CuO}_{4}$ film grown by ALL-MBE on an LSAO substrate, measured at three different frequencies, $20 \mathrm{kHz}$ (black), $40 \mathrm{kHz}$ (red), and $90 \mathrm{kHz}$ (blue). (b) The corresponding $\operatorname{Im} V_{p}(T)$ data. (c) The $\operatorname{Re} M(T)$ data from the same film, obtained by calibrating the raw data using the $\mathrm{Nb}$ measurements, and by normalizing the calibrated data using the corresponding data taken from a blank substrate. Inset of (c): a zoom-in at low temperature range, showing almost no difference among measurements made at different frequencies. (d) The corresponding $\operatorname{Im} M(T)$ data. The small differences near $T_{c}$ are intrinsic and they originate from the frequency dependence of $\hat{\sigma}(\omega)$ near the transition. Inset of (d): a zoom-in at low temperature range, showing that there is almost no difference among measurements made at different frequencies 

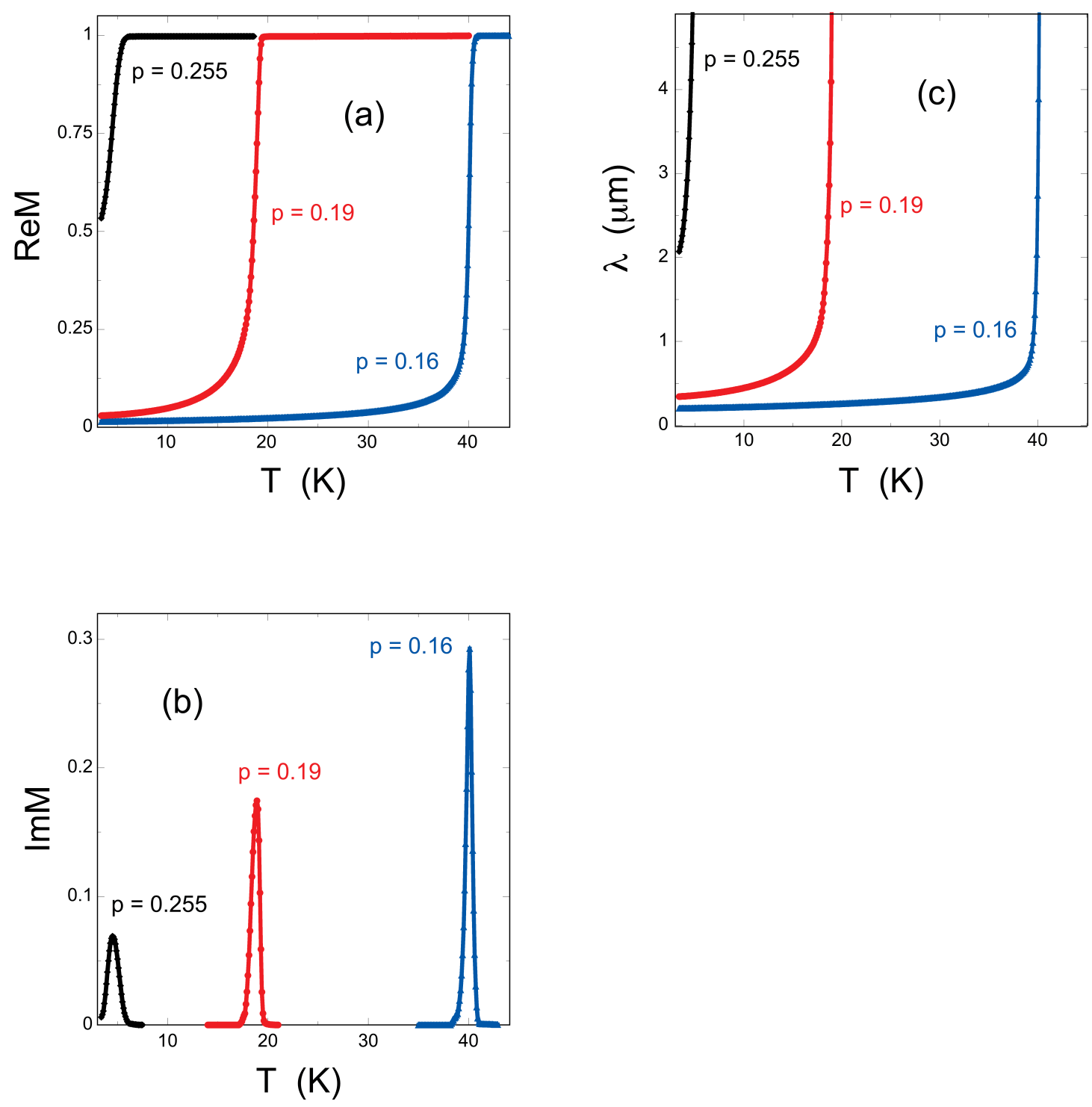

FIG. 5. (a) The ReM(T) data from three representative LSCO films grown by ALL-MBE on LSAO substrates: one optimally doped $\left(T_{c}=41 \mathrm{~K}, p=0.16\right)$, the second overdoped $\left(T_{c}=19.5 \mathrm{~K}, p=0.19\right)$, and the third extremely overdoped $\left(T_{c}=5.5 \mathrm{~K}, p=0.255\right)$. (b) The corresponding $\operatorname{Im} M(T)$ data for the same three films. (c) The $\lambda(T)$ dependences derived from the corresponding mutual inductance data. The data shown here were measured at $v=40$ $\mathrm{kHz}$, but the $\lambda(T)$ curves derived from the inductance data measured at $v=20 \mathrm{kHz}$ and $v=90 \mathrm{kHz}$ were essentially identical. 